\title{
基于石墨烯森林电极的摩擦纳米发电机
}

\author{
韩杰敏，王 梅，全召民，马一飞 \\ (山西大学 激光光谱研究所, 极端光学协同创新中心, 量子光学与光量子器件国家重点实验室, 太原 030006)
}

摘 要: 本研究提出并制备了一种以石墨烯森林为电极的摩擦纳米发电机。利用等离子体增强化学气相沉积工艺制 备了与其它墙形坚直薄片状的石墨烯形貌差异较大的石墨烯森林。此石墨烯森林具有低薄膜电阻((110 \pm 5$) \Omega / \square)$ 以及独特的离散“树状”结构, 有利于石墨烯森林与其他电极材料之间的接触和摩擦。根据石墨烯森林的形貌优势, 将聚酰亚胺膜和石墨烯森林膜作为电极组装摩擦纳米发电机, 其开路电压可达 $20 \mathrm{~V}$, 短路电流可达 $0.75 \mu \mathrm{A}$ 。此外, 还讨论了基于石墨烯森林的摩擦纳米发电机的工作原理。最后, 利用此纳米发电机点亮了演示电路中三个不同颜色 的 LED，证明了石墨烯森林在摩擦纳米发电机中的有效应用。

关 键 词: 石墨烯森林; 等离子体增强化学气相沉积; 摩擦纳米发电机 中图分类号: TM31 文献标识码: A

\section{Triboelectric Nanogenerator Based on Graphene Forest Electrodes}

\author{
HAN Jie-Min, WANG Mei, TONG Zhao-Min, MA Yi-Fei
}

(State Key Laboratory of Quantum Optics and Optics Quantum Devices, Collaborative Innovation Center of Extreme Optics, Institute of Laser Spectroscopy, Shanxi University, Taiyuan 030006, China)

\begin{abstract}
This study presents a triboelectric nanogenerator (TENG) with graphene forest as electrode. Graphene forest was fabricated by plasma enhanced chemical vapor deposition (PECVD) process, which shows great different morphology compared with other 'wall-like' vertically oriented graphene so far. Graphene forest films are composed of graphene nanoflakes, exhibiting not only low sheet resistance $((110 \pm 5) \Omega / \square)$ but also uniquely discrete 'tree-like' morphology, which are favorable to contact and friction with other materials. According to its morphology superiority, Kapton film and graphene forest film were utilized as electrodes for triboelectric nanogenerator, in which an open circuit voltage of $20 \mathrm{~V}$ and a short circuit current of $0.75 \mu \mathrm{A}$ are obtained. Furthermore, the working principle of the graphene forest based triboelectric nanogenerator was discussed. At last, three LEDs of different color in demo circuit were lighted up by as-prepared nanogenerator, which proves the effective application of graphene forest in triboelectric nanogenerator.
\end{abstract}

Key words: graphene forest; plasma enhanced chemical vapor deposition; triboelectric nanogenerator

石墨烯是一种二维结构材料，具有高电导率 ${ }^{[1]}$ 、 高比表面积 ${ }^{[2]}$ 和优异的机械性能 ${ }^{[3]}$ ，因而作为碳基 电极材料被广泛应用于透明电极 ${ }^{[4]}$ 、太阳能电池 ${ }^{[5]}$ 、
超级电容器 ${ }^{[6]}$ 以及锂离子电池 ${ }^{[7]}$ 。与传统的二维石 墨烯相比，竖直石墨烯(Vertically Oriented Graphene, VG)不但具备二维石墨烯的优异性能, 还具有裸露

收稿日期：2018-11-07; 收到修改稿日期：2018-12-19

基金项目：国家自然科学基金青年项目(21805174); 山西省百人计划青年项目; 山西省重点研发计划(国际科技合作)项目 (201803D421082); 山西省应用基础研究计划项目(201801D221100) National Natural Science Foundation of China (21805174); Shanxi Province 100-Plan Talents Program; Key Research and Development Program of Shanxi Province for International Cooperation (201803D421082); Applied Basic Research Project of Shanxi Province (201801D221100)

作者简介：韩杰敏(1992-)，男，硕士研究生. E-mail: jiemin.han@foxmail.com

通讯作者：马一飞, 讲师. E-mail: mayifei@sxu.edu.cn 
的尖锐边缘和非堆叠的形貌等优异特性 ${ }^{[8]}$, 在超级

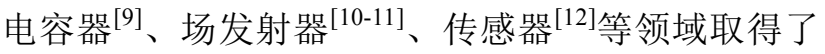
良好的应用效果。

目前, $V G$ 主要通过等离子体增强化学气相沉积 (Plasma Enhanced Chemical Vapor Deposition, PECVD)工艺实现制备。广泛报道的 VG 多为垂直于基

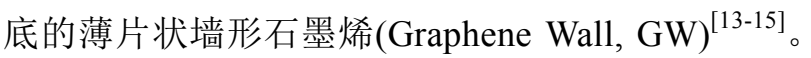
最近, 本课题组通过改变合成条件获得了一种新型 结构的 VG, 这种新型的 VG 具有 “森林” 状形貌, 所 以将其称为石墨烯森林(Graphene Forest, GF)。由于 GF 具有高比表面积和相对独立的坚直石墨烯单元 的优点, 可以在提供较大有效比表面积的同时表现 出良好的弯折耐受性 ${ }^{[16-17]}$ 。

基于摩擦起电效应的纳米发电机(Triboelectric Nanogenerator, TENG) 是将机械能转化为电能的有 效工具 ${ }^{[18]}$, 并可作为电源为设备提供电能 ${ }^{[18-19]}$, 有 巨大的应用潜力。TENG 具有输出电压高、绿色环 保、安全性好、体积质量小、成本低等优点 ${ }^{[20-21]}$ 。 在 TENG 的设计过程中, 电极材料的选择和形貌设 计是影响 TENG 性能的关键因素 ${ }^{[22-25]}$ 。三维结构的 GF 具有大的比表面积和优异的电荷传输能力, 而 且其特殊的三维坚直结构还可增加接触面积, 因此 可以作为 TENG 的优良电极, 但是目前尚未有 GF 用于 TENG 电极的报道。

本工作利用管式射频 PECVD 系统制备出形貌 均一、结构致密的“树状”结构的三维石墨烯森林并 将其应用于 TENG, 取得了良好的应用效果。本研 究可以为以后石墨烯在 TENG 方面的应用提供指 导作用。

\section{1 实验方法}

\section{1 石墨烯森林(GF)的制备}

实验选用 $5 \mathrm{~cm} \times 5 \mathrm{~cm}$ 的石英片作为生长基底。 首先依次使用去离子水和无水乙醇清洗石英片, 并 用氮气吹干。然后将清洗后的石英片放置于射频等 离子体增强化学气相沉积(RF-PECVD)系统的加热 区域中央, 如图 1 所示。将腔室抽真空至压力低于 $1 \mathrm{mTorr}$, 然后以 $8 \mathrm{sccm}$ 的流速通入氢气, 将腔室加 热至 $850{ }^{\circ} \mathrm{C}$ 并保持 $5 \mathrm{~min}$, 还原石英基底表面的氧 化物。而后调节射频电源的功率至 $200 \mathrm{~W}$, 电离氢 气产生等离子体, 保持 $2 \mathrm{~min}$, 进一步还原石英基底 表面的氧化物。然后以 3、1 $\mathrm{sccm}$ 的流速分别通入 乙炔、氢气，同时调节射频电源的功率为 $280 \mathrm{~W}$ 并 保持 $2 \mathrm{~h}$ 。生长结束后关闭射频电源, 停止通入气体 和加热, 让系统自然冷却至室温, 释压至大气压,

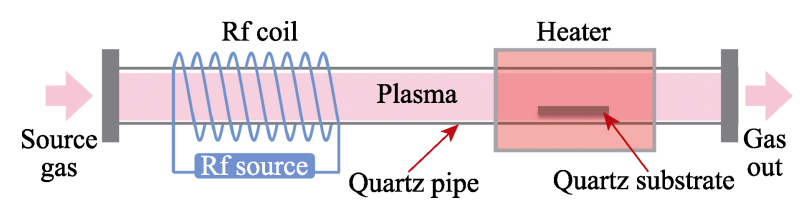

图 1 等离子体增强化学气相沉积(PECVD)系统的结构示意图 Fig. 1 Scheme of the plasema enhanced chemical vapor deposition system

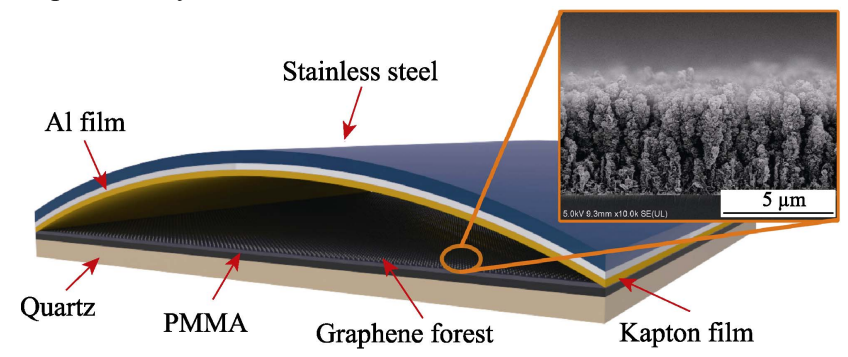

图 2 基于石墨烯森林的摩擦纳米发电机的结构示意图

Fig. 2 Schematic diagram of GF-based triboelectric nanogenerator

得到 $\mathrm{GF}$ 样品。

\section{2 摩擦纳米发电机(TENG)的制备}

图 2 为基于 GF 电极的 TENG 的结构示意图。 聚酰亚胺(Kapton)为负极材料, GF 为正极材料。在 正负极材料接触过程中, Kapton 可以从 GF 中获得 电子。在 Kapton 薄膜上蒸镀一层厚度为 $150 \mathrm{~nm}$ 的 铝薄膜作为导电电极, 然后将 $\mathrm{Al} /$ Kapton 薄膜附着 在弯曲的不锈钢板上形成拱形结构。不锈钢板具有 弹性, 可弯曲, 变形后可自动恢复。配制浓度为 $46 \mathrm{mg} / \mathrm{mL}$ 的聚甲基丙烯酸甲酯(poly(methyl methacrylate), PMMA)氯苯溶液, 取 $5 \mathrm{~mL}$ 旋涂于 GF 的表 面(旋转速度为 $5000 \mathrm{r} / \mathrm{min}$, 持续 $60 \mathrm{~s}$, 在室温下干 燥 $10 \mathrm{~min}$ )。此 PMMA 将填充于 GF 的空隙中, 进一 步增强 GF 的摩擦及坚直方向的弯曲耐受性。

\section{3 材料表征}

通过场发射扫描电子显微镜(FE-SEM, Hitachi, SU8010)和高分辨透射电子显微镜(HRTEM，JEOL, JEM-2100F)表征 GF 样品的形貌; 利用拉曼光谱 (Renishaw, RM-1000 Invia)分析样品的碳键结构, 波 长为 $532 \mathrm{~nm}$ ( $\mathrm{Ar}^{+}$离子激光); 在室温下用四点探针 法(Keithley 2420I-V)测量样品不同位置处的薄膜电 阻并取平均值; 利用力刺激器(Z-TEC ZPS 100)快速 挤压 TENG, 并使用示波器(Tektronix DPO 3052)和 皮安表(Keithley 6485)测量其开路电压和短路电流。

\section{2 结果与讨论}

\section{1 石墨烯森林的结构与形貌分析}

图 3(a) (b) 是 GF 的 SEM 侧视照片, 石墨烯森 

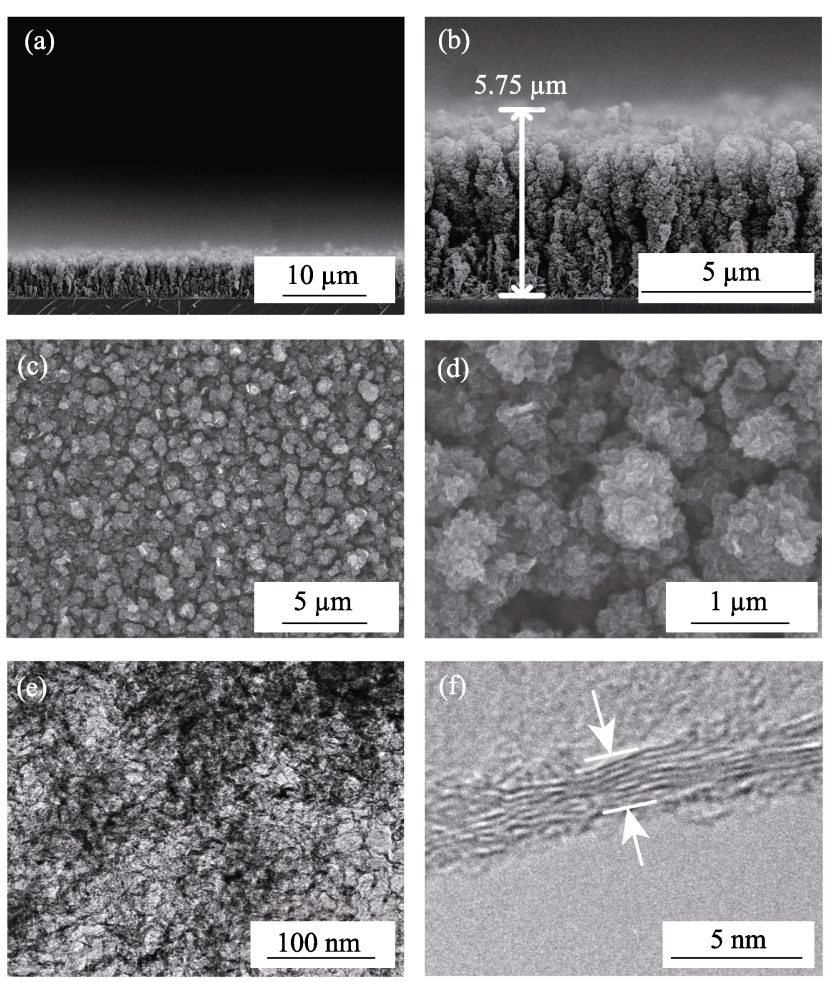

图 3 石英基底上 GF 侧视(a) (b) 和顶视(c) (d) SEM 照片, 以及 GF 的高分辨 TEM 照片(e) (f)

Fig. 3 SEM images of graphene forest on a quartz substrate from side-view (a-b) and top-view (c-d), and HRTEM images of graphene forest in different magnifications (e-f)

林表现出了不同于其他坚直石墨烯的形貌 ${ }^{[8]}$ 。微观 下每一支坚直的石墨烯结构类似于一棵“树”，石墨 烯薄膜整体如同一片茂密的“森林”，石墨烯“树”,高 度均匀, 约为 $5.75 \mu \mathrm{m}$, “树”间距离约为 $300 \mathrm{~nm}$ 。 图 3(c) (d)展示了 GF 的 SEM 顶视照片, 每一个 “树状”, 石墨烯结构彼此相互接近, 排列紧密。如 图 3(e) (f)的 HRTEM 所示, 每棵石墨烯 “树” 是由 石墨烯片随机叠加而成的, 且每个石墨烯纳米片由 多层石墨烯组成。

拉曼光谱是一种重要的检测方法, 广泛用于揭 示石墨烯的质量和估算石墨烯的层数 ${ }^{[26]}$ 。GF 的拉曼 光谱如图 4 所示, 可以看到主要有 $\mathrm{D}$ 峰 $\left(\sim 1350 \mathrm{~cm}^{-1}\right)$ 、 $G$ 峰 $\left(\sim 1580 \mathrm{~cm}^{-1}\right)$ 和 $2 \mathrm{D}$ 峰( $\left.2700 \mathrm{~cm}^{-1}\right)$ 。通常, D 峰 与六原子环的呼吸振动有关, 是由晶格振动离开布 里渊区中心引起的, 通常被认为是石墨烯的无序 振动峰, 用于表征石墨烯样品中的结构缺陷或边 缘 ${ }^{[26-27]}$ 。在 GF 的拉曼光谱中, 有非常强的 D 峰, 这 主要有两个方面的原因: 一方面, 通过 PECVD 生 长的 $\mathrm{GF}$ 不是由完整的单晶石墨烯构成, 而是由许 多具有裸露尖锐边缘的石墨烯纳米片堆叠构成, 从 而导致 D 峰较高。另一方面, 等离子体中的一些阳 离子和氢激子(H-radicals)会对石墨烯表面产生刻蚀

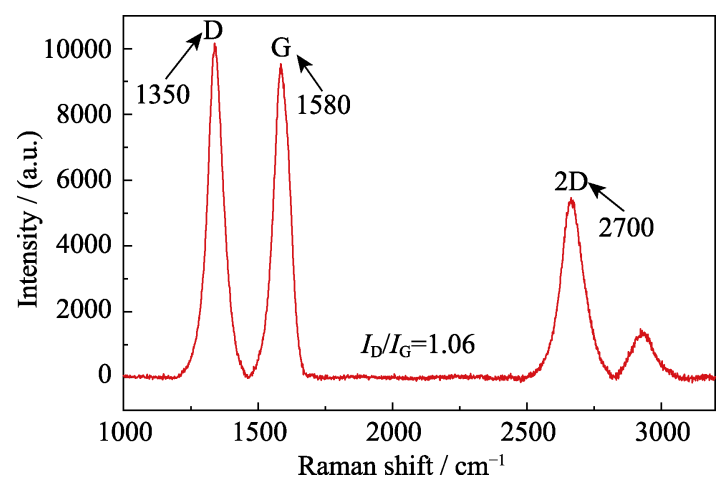

图 4 石墨烯森林的拉曼光谱图

Fig. 4 Raman spectrum of the graphene forest

作用，造成缺陷，同样会导致 D 峰升高。G 峰与 iTO 和 LO 声子的振动有关, 是由于环和链中 $\mathrm{sp}^{2}$ 碳键的 拉伸振动而产生的，具有 $\mathrm{E}_{2 \mathrm{~g}}$ 对称性，可反映石墨烯 片层数 ${ }^{[26-27]}$ 。2D 峰源于两个 $\mathrm{iTO}$ 声子二阶振动过 程，用于表征石墨烯中碳原子的层间堆垛方式。一 般用 $\mathrm{G}$ 峰与 $2 \mathrm{D}$ 峰的强度比 $\left(I_{2 \mathrm{D}} / I_{\mathrm{G}}\right)$ 来判断石墨烯层 数 ${ }^{[27-28]}$, 但由于 $\mathrm{GF}$ 独特的坚直形貌, 很难通过拉 曼光谱中的 $I_{2 \mathrm{D}} / I_{\mathrm{G}}$ 来估计石墨烯的层数。由 TEM 照 片(图 3(f)) 可以看出每个坚直的石墨烯纳米片是由 2 8 层石墨烯组成。

导电性是石墨烯在电子器件应用中的一项重要 性能。由于 GF 独特的形貌，其电阻值不仅与石墨烯 的质量有关，而且与石墨烯的结构有关。经过对样 品不同点处进行测量, 得到 $\mathrm{GF}$ 薄膜的平均薄膜电 阻为 $(110 \pm 5) \Omega / \square$ 。由于 GF 具有独特的“树状”结 构，每个 GF 纳米片之间相互交叉，形成一种立体的 导电网络, 有效降低了 $\mathrm{GF}$ 的整体电阻。因此 GF 表 现出较低的薄膜电阻。

\section{2 摩擦纳米发电机的原理与性能表征}

基于 GF 电极的 TENG 的工作原理如图 5(a)所 示。最初, 在没有任何压力的情况下, Kapton 薄膜和 $\mathrm{GF}$ 表面无电荷。当垂直压力施加到 TENG 的顶部 后, Kapton 薄膜与 $\mathrm{GF}$ 相互靠近并接触，电子从 $\mathrm{GF}$ 转移到 Kapton 薄膜表面使其带负电, 相应的 GF 由 于电子流失而带正电。由于产生的表面电荷几乎都 在同一个平面, 在 Kapton 薄膜与 GF 之间产生的静 电电位差很小, 无电信号。当压力释放时, 装置将恢 复到原来的位置。Kapton 薄膜和 GF 表面分离, 在 Kapton 薄膜和 GF 之间就会产生一个较大的电位 差。为了达到平衡, 电子开始通过外部电路从铝电 极顶部流向 GF 电极, 从而产生电信号, 直到装置完 全恢复平衡。平衡时没有电信号。当压力再次作用 于 TENG 时, Kapton 薄膜再次靠近并接触 GF 使得 平衡再次被打破, 静电电位开始减小, 电子通过外 
(a)

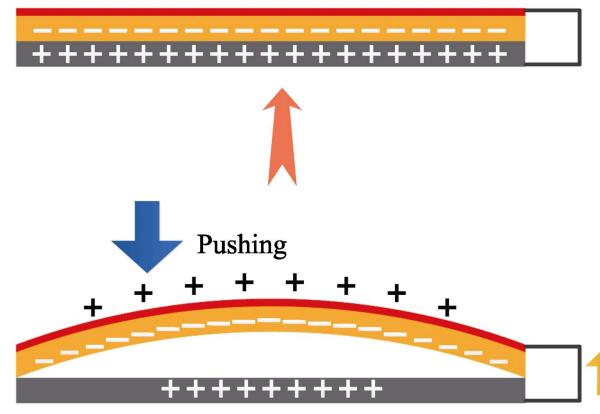

(b)

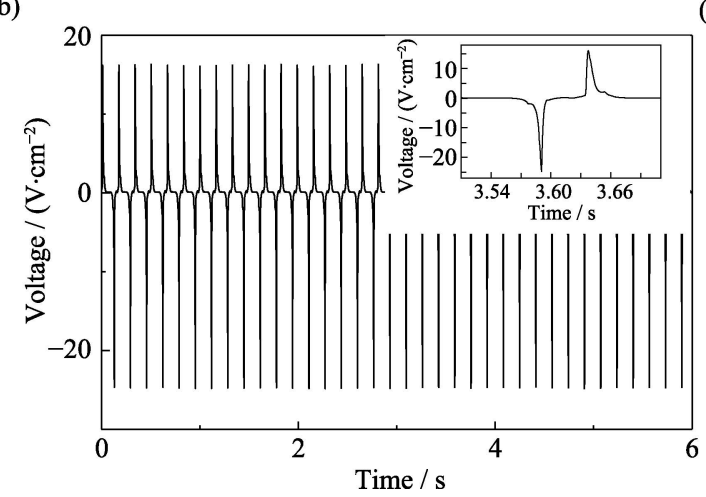

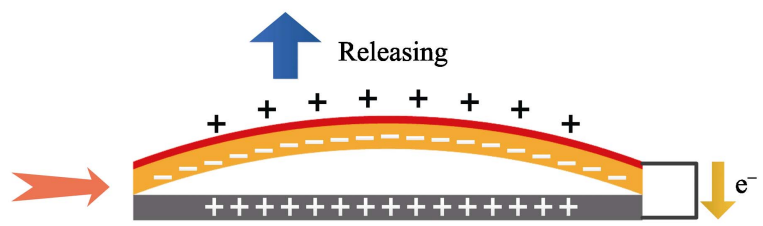

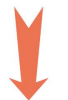

Aluminum
Kapton

GF

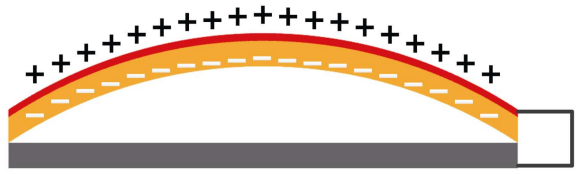

(c)

图 5 基于石墨烯森林的摩擦纳米发电机的工作原理(a); 基于石墨烯森林的摩擦纳米发电机的 开路电压输出(b)和短电流输出(c), 插图为其单一周期的曲线

Fig. 5 Working mechanism of GF based TENG (a), open circuit voltage output (b) and short circuit current output (c) of graphene forest based triboelectric nanogenerator with insets showing the representative cycle of each plot

部电路反向流动, 从而产生电信号, 直到设备被完 全按压达到另一种平衡, 在这个按压与释放的循环 中, 电子以相反的方向在外部电路流过两次, 从而 形成交流电信号。

为了解基于 GF 的 TENG 的性能, 本课题组对 其进行了开路电压和短路电流测试, 如图 5(b) (c) 所示。 $1 \mathrm{~cm}^{2}$ 大小的 TENG 在测试过程中可以产生 稳定的电压和电流输出, 分别为 $20 \mathrm{~V}$ 和 $0.75 \mu \mathrm{A}$ 。 图 5(b)中的插图为 TENG 在一个周期(3.51 3.71 s) 内开路电压的分布情况，3.5906 $\mathrm{s}$ 时电压处于波谷 $\left(-24.75495 \mathrm{~V} / \mathrm{cm}^{2}\right), 3.6374 \mathrm{~s}$ 时电压处于波峰 $\left(16.07955 \mathrm{~V} / \mathrm{cm}^{2}\right)$; 图 5(c)中的插图为 $\mathrm{TENG}$ 在一个 周期 $(0.41 \sim 0.58 \mathrm{~s})$ 内短路电流的分布情况, $0.4599 \mathrm{~s}$ 时电流处于波谷 $\left(-0.7571 \mu \mathrm{A} / \mathrm{cm}^{2}\right), 0.5065 \mathrm{~s}$ 时电流 处于波峰 $\left(0.4112 \mu \mathrm{A} / \mathrm{cm}^{2}\right)$ 。表明基于 GF 的 TENG 的电压和电流响应很快。

此外, 为了进一步展示 TENG 的发电性能, 将 TENG 与整流器、不同颜色的 LED 分别串联成闭合 回路, 在按压 TENG 的过程中, 产生的电流经过整 流后能够点亮不同颜色的 LED(见图 6), 表明 TENG 具有独立驱动 LED 的能力。
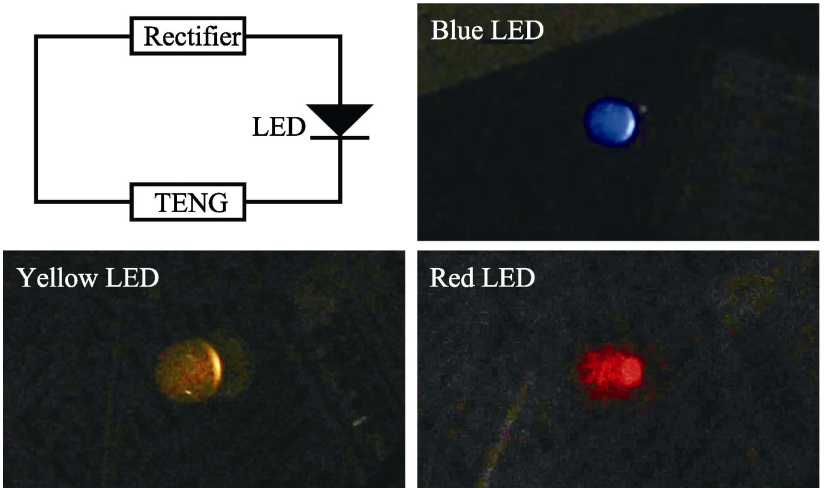

图 6 基于 GF 的 TENG 点亮不同颜色的 LED

Fig. 6 LEDs of different color powered by graphene forest based triboelectric nanogenerator with a simple circuit

\section{3 结论}

本研究通过等离子体化学气相沉积工艺制备出 具有高比表面积、相对独立坚直石墨烯单元、低薄 膜电阻 $((110 \pm 5) \Omega / \square)$ 的石墨烯森林，是一种优良 的电极材料。更重要的是, 基于石墨烯森林电极构 建的摩擦纳米发电机具有稳定的输出电压 $(20 \mathrm{~V})$ 和 电流 $(0.75 \mu \mathrm{A})$ ，表现出良好的应用效果。 


\section{参考文献:}

[1] DE S, COLEMAN J N. Are there fundamental limitations on the sheet resistance and transmittance of thin graphene films? ACS Nano, 2010, 4(5): 2713-2720.

[2] ZHU Y, MURALI S, STOLLER M D, et al. Carbon-based supercapacitors produced by activation of graphene. Science, 2011, 332(6037): 1537-1541.

[3] CHEN Z, REN W, GAO L, et al. Three-dimensional flexible and conductive interconnected graphene networks grown by chemical vapour deposition. Nature Materials, 2011, 10(6): 424-428.

[4] GUO Y, LIN L, ZHAO S, et al. 2D hybrid nanostructured dirac materials for broadband transparent electrodes. Advanced Materials, 2015, 27(29): 4315-4321.

[5] VAZQUEZMENA O, BOSCO J P, ERGEN O, et al. Performance enhancement of a graphene-zinc phosphide solar cell using the electric field-effect. Nano Letters, 2014, 14(8): 4280-4285.

[6] $\mathrm{BO}$ Z, ZHU W, MA W, et al. Vertically oriented graphene bridging active-layer/current-collector interface for ultrahigh rate supercapacitors. Advanced Materials, 2013, 25(40): 5799-5806.

[7] CHANG J, HUANG X, ZHOU G, et al. Multilayered Si nanoparticle/ reduced graphene oxide hybrid as a high-performance lithium-ion battery anode. Advanced Materials, 2014, 26(5): 758-764.

[8] BO Z, YANG Y, CHEN J, et al. Plasma-enhanced chemical vapor deposition synthesis of vertically oriented graphene nanosheets. Nanoscale, 2013, 5(12): 5180-5204.

[9] SHENG L, WEI T, LIANG Y, et al. Vertically oriented graphene nanoribbon fibers for high-volumetric energy density all-solid-state asymmetric supercapacitors. Small, 2017, 13(22): 1700371-1-9.

[10] BELLUCCI S, MALESEVIC A, MICCIULLA F, et al. Comparative field emission from vertically aligned few-layer graphene and carbon nanotubes. Nanoscience and Nanotechnology Letters, 2011, 3(6): 907-912.

[11] MALESEVIC A, KEMPS R, VANHULSEL A, et al. Field emission from vertically aligned few-layer graphene. Journal of Applied Physics, 2008, 104(8): 084301-1-6.

[12] MAO S, YU K, CHANG J, et al. Direct growth of verticallyoriented graphene for field-effect transistor biosensor. Scientific Reports, 2013, 3: 1696-1-6.

[13] ZHU M Y, OUTLAW R A, BAGGE-HANSEN M, et al. Enhanced field emission of vertically oriented carbon nanosheets synthesized by $\mathrm{C}_{2} \mathrm{H}_{2} / \mathrm{H}_{2}$ plasma enhanced CVD. Carbon, 2011, 49(7): 2526-2531.

[14] SHUAI X, BO Z, KONG J, et al. Wettability of vertically-oriented graphenes with different intersheet distances. RSC Advances, 2017, 7(5): $2667-2675$.
[15] OUYANG B, ZHANG Y, ZHANG Z, et al. Green synthesis of vertical graphene nanosheets and their application in highperformance supercapacitors. RSC Advances, 2016, 6(28): 2396823973.

[16] MA Y, WANG M, KIM N, et al. A flexible supercapacitor based on vertically oriented 'graphene forest' electrodes. Journal of Materials Chemistry A, 2015, 3(43): 21875-21881.

[17] WANG M, MA Y. Nitrogen-doped graphene forests as electrodes for high-performance wearable supercapacitors. Electrochimica Acta, 2017, 250: 320-326.

[18] WANG Z L. Triboelectric nanogenerators as new energy technology for self-powered systems and as active mechanical and chemical sensors. ACS Nano, 2013, 7(11): 9533-9557.

[19] ZHU G, PAN C, GUO W, et al. Triboelectric-generator-driven pulse electrodeposition for micropatterning. Nano Letters, 2012, 12(9): 4960-4965.

[20] TANG W, JIANG T, FAN F R, et al. Liquid-metal electrode for high-performance triboelectric nanogenerator at an instantaneous energy conversion efficiency of $70.6 \%$. Advanced Functional Materials, 2015, 25(24): 3718-3725.

[21] ZHU G, LIN Z H, JING Q, et al. Toward large-scale energy harvesting by a nanoparticle-enhanced triboelectric nanogenerator. Nano Letters, 2013, 13(2): 847-853.

[22] HE X, ZI Y, GUO H, et al. A highly stretchable fiber-based triboelectric nanogenerator for self-powered wearable electronics. Advanced Functional Materials, 2017, 27(4): 1604378-1-8.

[23] PU X, LIU M, CHEN X, et al. Ultrastretchable, transparent triboelectric nanogenerator as electronic skin for biomechanical energy harvesting and tactile sensing. Science Advances, 2017, 3(5): e1700015-1-11.

[24] KIM S, GUPTA M K, LEE K Y, et al. Transparent flexible graphene triboelectric nanogenerators. Advanced Materials, 2014, 26(23): 3918-3925.

[25] YANG J, LIU P, WEI X, et al. Surface engineering of graphene composite transparent electrodes for high performance flexible triboelectric nanogenerators and self-powered sensors. ACS Applied Materials \& Interfaces, 2017, 9(41): 36017-36025.

[26] FERRARI A C, BASKO D M. Raman spectroscopy as a versatile tool for studying the properties of graphene. Nature Nanotechnology, 2013, 8(4): 235-246.

[27] MALARD L M, PIMENTA M A A, DRESSELHAUS G, et al. Raman spectroscopy in graphene. Physics Reports, 2009, 473(5/6): 51-87.

[28] REINA A, JIA X, HO J, et al. Large area, few-layer graphene films on arbitrary substrates by chemical vapor deposition. Nano Letters, 2009, 9(1): 30-35. 